\title{
The Value of High Resolution Computed Tomography in the Diagnostics of Small Opacities and Complications of Silicosis in Mine Machinery Manufacturing Workers, Compared to Radiography
}

\author{
Jinkai Sun ${ }^{1,2}$, Dong Weng ${ }^{1}$, Changshan $\mathrm{JIN}^{1}$, Bo Y $\mathrm{AN}^{2}$, Guihua XU ${ }^{2}$, Bo JiN ${ }^{2}$, \\ Shenning $\mathrm{XIA}^{2}$ and Jie CHEN ${ }^{1}$
}

${ }^{1}$ Division of Pneumoconiosis, School of Public Health, China Medical University and ${ }^{2}$ Shenyang No.9 Hospital, China

\begin{abstract}
The Value of High Resolution Computed Tomography in the Diagnostics of Small Opacities and Complications of Silicosis in Mine Machinery Manufacturing Workers, Compared to Radiography: Jinkai Sun, et al. Division of Pneumoconiosis, School of Public Health, China Medical University, China-The purpose of this study was to assess the value and usefulness of high resolution computed tomography (HRCT) in the diagnostics of small opacities and complications of silicosis in mine machinery manufacturing workers, compared to conventional radiograms. The diagnosis of silicosis is mainly based on the radiological findings of workers exposed to the dust causing lung fibrosis. However, on radiograms many parenchymal structures overlap, which limits the sensitivity and specificity of the method. Difficulties in accurate interpretation of conventional radiograms in silicosis also result from their relatively low resolution. We randomly selected 30 I period silicosis patients from 77 I period silicosis patients working at a mine machinery manufacturing company. Out of 1078 non-silicosis and $1620^{+}$period silicosis subjects, 30 non-silicosis and $300^{+}$period silicosis subjects were randomly selected and matched with the 30 I period silicosis subjects by age $( \pm 3.0 \mathrm{yr})$ and occupational exposure time $( \pm 2.0 \mathrm{yr})$. Chest $X-$ rays were taken at maximal inspiration. For the HRCT examination, the GE Somatom plus apparatus was used. Eight, five and six subjects were respectively diagnosed as $\mathrm{O}^{+}$, I or $\mathrm{I}^{+}$period silicosis based on HRCT
\end{abstract}

Received Feb 2, 2008; Accepted Jun 2, 2008

Published online in J-STAGE Jul 29, 2008

Correspondence to: J. Chen, Division of Pneumoconiosis, School of Public Health, China Medical University, 92 North 2nd Road, Heping District, Shenyang 110001, China

(e-mail: chenjie@mail.cmu.edu.cn) among 90 subjects whose original diagnoses were nonsilicosis, $0^{+}$or I period silicosis based on radiography. The numbers of small opacities in HRCT scans were significantly higher than those seen in radiography in all lung zones $(p<0.01)$. HRCT was more sensitive than radiography in detecting small opacities of midout zones of the lung, but no statistical significance was found between the two methods in the detection of small opacities of lower zones of the lung. A statistically significant increase in the detectability of bulla, emphysema, pleural, mediastinal and hilar changes was observed $(p<0.05)$. HRCT might be more sensitive than radiography in detecting lung parenchymal changes suggestive of silicosis.

(J Occup Health 2008; 50: 400-405)

Key words: Silicosis, High resolution computed tomography, Radiography

Silicosis is a pulmonary disease contracted through the inhalation of crystalline silica, which induces a fibrogenic tissue response. The most usual form of silicosis occurs after many years of exposure to relatively low levels of dust. Silicosis is characterized by the presence of small discrete nodules ${ }^{1-3)}$.

The diagnosis of silicosis is mainly based on radiological findings, according to diagnosis standard GBZ70-2002 of pneumoconiosis and diagnosis standard films of pneumoconiosis in China ${ }^{4)}$. One of the most commonly encountered changes in the picture of simple pneumoconiosis is nodules. However, individual variable sensitivity to silica exposure and the individual variable responses of the lungs to its presence hinder early detection of pneumoconiosis in the subclinical stage, as well as later prognosis of the disease. The evaluation of $\mathrm{X}$-ray changes and functional respiratory disorders in 
some cases of initial reticular forms of pneumoconiosis is difficult ${ }^{5-7)}$. Therefore, new techniques of visualization of the early changes, which could make the diagnosis of pneumoconiosis at subclinical stages easier and prognosis of the progression of the illness (due to observation of the present changes), are necessary ${ }^{8}$. A number of attempts have been made to assess and compare various radiological and functional methods for pneumoconiosis. Some scholars have compared X-ray changes after HRCT of the chest with the findings of post mortem pathohistological examinations ${ }^{9-12)}$. Computer tomography (CT) has an increasing role in the radiological evaluation of pneumoconiosis ${ }^{9,10)}$. HRCT scans at the early stage of silicosis demonstrate irregular nonseptal small linear opacities in addition to nodular opacities diffusely distributed throughout both lungs ${ }^{10)}$. An HRCT scan is more sensitive than chest radiography in detecting lung parenchymal changes suggestive of silicosis and early confluence of small opacities in the lung ${ }^{11,12)}$. Its significance for patients with silicosis has not been definitively established in the current literature and it is not currently accepted as a diagnostic tool for the detection of the disease.

This study aimed to assess the diagnostic value of HRCT for silicosis. We randomly selected 90 workers occupationally exposed to silica dust in Shenyang Minemachine Manufacture Company. Thirty male nonsilicosis subjects and 30 male $0^{+}$period silicosis subjects were matched with the 30 male I period silicosis patients by age and occupational exposure time. By comparison of radiography and HRCT scans on small opacity and complications in silicosis subjects, we expect to provide useful information and proof for the better and earlier diagnosis of silicosis.

\section{Subjects and Methods}

\section{Subjects}

In Shenyang Mine-machine Manufacture Company, we randomly selected 30 I period silicosis patients from 77 I period silicosis patients in whom silicosis was confirmed based on their occupational history and radiological alterations compatible with the disease. Out of the 1,078 non-silicosis and $1620^{+}$period silicosis subjects, 30 nonsilicosis and $300^{+}$period silicosis subjects were randomly selected and matched with the 30 I period silicosis subjects by age $( \pm 3.0 \mathrm{yr})$ and occupational exposure time $( \pm 2.0 \mathrm{yr})$. All subjects were male and engaged in sand casting. The age of the non-silicosis group ranged from $32.0-72.0 \mathrm{yr}[48.0 \pm 5.2($ mean $\pm \mathrm{SD}) \mathrm{yr}]$. The exposure time ranged from 10.0-35.0 yr [15.7 $\pm 2.8($ mean \pm SD) $\mathrm{yr}$. The age of the $0^{+}$period silicosis group ranged from $35.0-68.0$ yr $[50.0 \pm 4.9($ mean $\pm \mathrm{SD}) \mathrm{yr}]$. The exposure time ranged from 10.5-33.0 yr [16.8 $\pm 3.2($ mean \pm SD) $\mathrm{yr}$. The age of I period silicosis group ranged from 34.0$70.0 \mathrm{yr}[51.0 \pm 4.8($ mean $\pm \mathrm{SD}) \mathrm{yr}]$. The exposure time ranged from $12.0-35.0 \mathrm{yr}[15.7 \pm 2.8($ mean $\pm \mathrm{SD}) \mathrm{yr}]$.

\section{Radiography and HRCT examinations}

Chest X-rays were taken using a standard Shimadzu Corporation $800 \mathrm{~mA}$ ED150 Rontgen unit, using settings of $120-140 \mathrm{kV}, 100 \mathrm{~mA}$ and a $0.1 \mathrm{~s}$ acquisition time. The subjects were placed in the dorsaventral position, and the distance between the focal plane and the film was $180 \mathrm{~cm}$. For the HRCT examination a GE Somatom plus apparatus, equipped with a special programme for high resolution algorithm image reconstruction was used. HRCT images of $2 \mathrm{~mm}$ slice thickness were obtained at $10-\mathrm{mm}$ intervals, using settings of $120 \mathrm{kV}, 300 \mathrm{~mA}$ and a $0.5 \mathrm{~s}$ acquisition time. HRCT scans were obtained during suspended deep inspiration in the supine position. Two window systems were used: pulmonary and supplementary, and mediastinal.

\section{Radiography and HRCT image reading and diagnosis}

Both chest radiographs and HRCT images were read blind according to the GBZ70-2002 standard by five independent readers who were members of Shenyang municipal pneumoconiosis diagnosis committee. The readers graded the severity of disease in each of the six zones of each pair of lungs. The profusion of small opacities for each radiograph was taken to be the median of the five readings. The presence of large opacities or other complications, such as tuberculosis, pleural thickening, and emphysema, was recorded in a similar fashion. The result reported was the consensus when three or more of the five readers' findings were in agreement. The diagnosis standard GBZ70-2002 of pneumoconiosis and diagnosis standard films of pneumoconiosis were used for the classification and comparative interpretation of X-rays and HRCT.

According to the diagnosis standard GBZ70-2002, the definition of non-silicosis is that there is no manifestation on X-rays. The definition of $0^{+}$is that the manifestation on X-rays is not enough to be diagnosed as I silicosis. The definition of I period silicosis is that the total profusion of small opacities is grade 1 and the distribution range reaches at least two lung zones. The definition of $\mathrm{I}^{+}$period silicosis is that the total profusion of small opacities is grade 1 and the distribution range exceeds four lung zones, or the total profusion of small opacities is grade 2 and the distribution range reaches four lung zones. The diagnosis of silicosis was mainly based on numbers of small opacities and numbers of lung zones by profusion of small opacities. We made a comparison of the radiography and HRCT images of non-silicosis, $0^{+}$ and I silicosis groups and analyzed numbers of small opacities and numbers of lung zones by profusion of small opacities to give an initial evaluation of HRCT's diagnostic value in the diagnostics of small opacities of silicosis. We also analyzed complications of silicosis such 
Table 1. Comparison of radiography and HRCT scans in diagnosis of silicosis of different groups

\begin{tabular}{lccccc}
\hline Group & \multicolumn{4}{c}{ Diagnosis } & Total \\
\cline { 2 - 5 } & 0 & $0^{+}$ & $\mathrm{I}$ & $\mathrm{I}^{+}$ & \\
\hline Radiography & 30 & 30 & 30 & 0 & 90 \\
HRCT & 22 & 33 & 29 & 6 & 90 \\
\hline
\end{tabular}

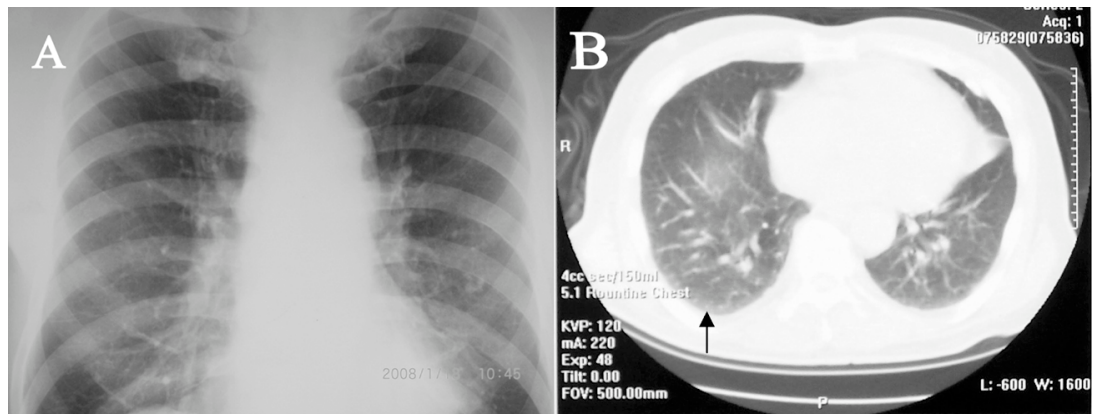

Fig. 1. A: There were no small opacities in the chest radiograph. The subject was originally diagnosed as non-silicosis based on radiography; B: HRCT scan displayed small numbers of opacities in the outer zone of the right lower lung (arrow). This subject could be diagnosed as $0^{+}$period silicosis based on HRCT scans.

as emphysema, pleura changes and tuberculosis, etc.

\section{Statistical analysis}

We used SPSS 11.5 software to conduct statistical analyses. Complications of silicosis, the number and perfusion of small opacities between chest radiograph and HRCT images were checked using the $\chi^{2}$ test. $p<0.05$ was considered statistically significant.

\section{Results}

Comparison of radiography and HRCT scans of small opacity in silicosis subjects

Eight subjects were diagnosed as $0^{+}$period silicosis based on HRCT of 30 subjects whose original diagnoses were non-silicosis based on radiography (Table 1). No small opacities were found in chest radiographs, but 8 subjects could be diagnosed as $0^{+}$period silicosis in HRCT scans. Three subjects' HRCT scans displayed small numbers of small opacities in the outer zone of the lower lung, and they could be diagnosed as $0^{+}$period silicosis (Fig. 1). There were small opacities in the mid-out zone of the right lower lung in 3 subjects' HRCT scans. Two subjects' HRCT scans showed profuse small opacities in their mid-out zones of the left lower lung.

Among the 30 subjects who were diagnosed as $0^{+}$ period silicosis, 5 subjects could be diagnosed as I period silicosis according to their HRCT scans (Table 1). One subject's chest radiograph showed that the small opacities were diffused in the outer zones of both lower lungs. He couldn't be diagnosed as I period silicosis according to the radiographic diagnosis, but the HRCT scans showed that the profusion of small opacities was consistent with I period silicosis's standard (Fig. 2). Two subjects' chest radiographs showed that the profusion in the lower zones of the right lung was corresponded to the diagnostic standard while the profusion in the lower zones of the left lung wasn't consistent with the standard. However, both subjects' HRCT scans revealed that the profusions in both lower zones of the lung were consistent with the diagnostic standard. The profusion of small opacities in their lower zones but not the middle zones of the right lungs conformed to the diagnostic standards, but they both conformed to the standards in HRCT scans.

Six subjects' who were I period silicosis based on radiographs were consistent with the diagnostic standards of $\mathrm{I}^{+}$period silicosis in HRCT scans (Table 1). There were large numbers of small opacities in the outer zones of both lower lungs of 2 subjects' radiographs (Fig. 3), and these findings were also demonstrated in mid-upper zones of the left lungs in HRCT scans. These 2 subjects could be diagnosed as $\mathrm{I}^{+}$period silicosis based on HRCT scans. There were profuse small opacities in the outer zones of both lower lungs of 4 subjects' radiographs, and these findings were also demonstrated in mid-upper zones 


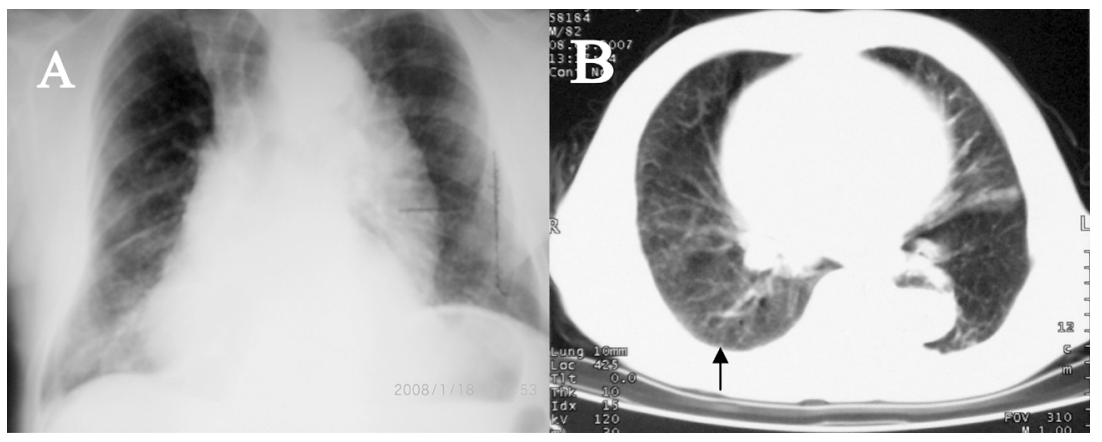

Fig. 2. A: This $0^{+}$silicosis subject's chest radiograph showed that the small opacities were diffused in the outer zones of both lower lungs. The subject couldn't be diagnosed as I period silicosis according to radiography diagnosis; B: HRCT scan displayed numbers of opacities in the outer zones of both lower lungs (arrow). The HRCT scans showed that the profusion of small opacities was consistent with the I period silicosis standard.
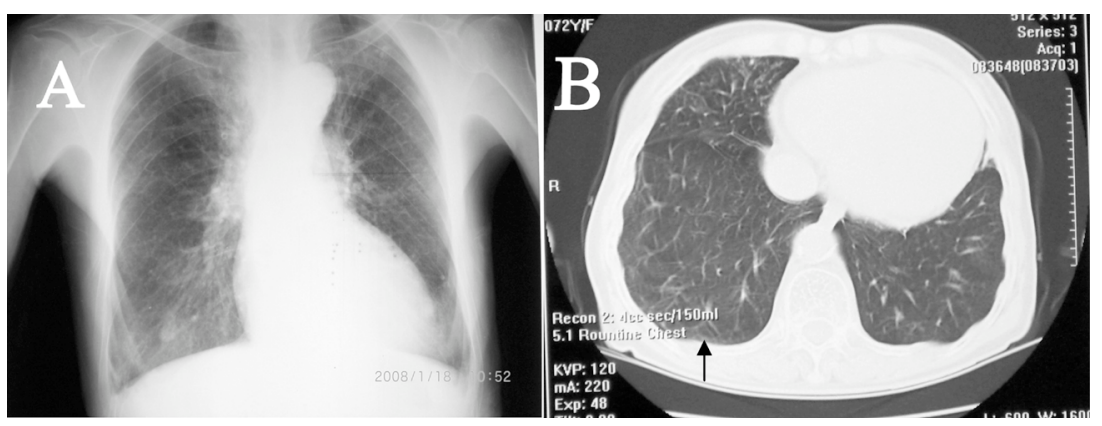

Fig. 3. A: This I period silicosis subject's chest radiograph showed that profuse small opacities were diffused in the outer zones of both lower lungs. The subject couldn't be diagnosed as $\mathrm{I}^{+}$period silicosis according to radiography diagnosis; B: HRCT scan displayed large numbers of opacities in the outer zones of both lower lungs (arrow). The HRCT scans showed that the profusion of small opacities was consistent with the $\mathrm{I}^{+}$period silicosis standard.

of right lungs in HRCT scans. These 2 subjects could be diagnosed as $\mathrm{I}^{+}$period silicosis based on HRCT scans.

Comparison of radiography and HRCT scans on numbers of small opacities and numbers of lung zones by profusion of small opacities

The distribution of the small opacities in double upper lung zones in HRCT scans was more profuse than that in radiography in a comparison of 90 subjects' radiographs and HRCT scans. In double lower lung zones, the changes were mainly thick linear fibrosis including small opacities in HRCT scans, while the changes were mainly small opacities on radiographs. The numbers of small opacities in HRCT scans were significantly higher than those on radiography in all lung zones (Table $2, \chi^{2}=15.783$, $p=0.007$ ).

Each subject has 6 lung zones, so there were 540 lung zones in the 90 subjects. The lung zones in HRCT scans were divided based on the location of HRCT and radiographs. There were more lung zones where the profusion $0-1$ and 1 of small opacities had diagnostic value in HRCT scans than that in radiographs, but no statistical significance was found (Table $3, \chi^{2}=4.173$, $p=0.124)$. According to the comparison of radiography and HRCT scans on numbers of small opacities and numbers of lung zones by profusion of small opacities, HRCT may be more sensitive than radiography at detecting the abnormal lung zones.

Comparison of radiography and HRCT scans on complications of silicosis

Twenty-six cases of bulla were detected in HRCT scans. However, only 5 cases of bulla were detected in radiographs. The detection rates of emphysema and bulla 
Table 2. Comparison of radiography and HRCT scans of numbers of small opacities of different groups

\begin{tabular}{|c|c|c|c|c|c|c|c|c|c|c|c|c|c|c|c|c|c|c|}
\hline \multirow[t]{3}{*}{ Group } & \multicolumn{9}{|c|}{ Right Lung } & \multicolumn{9}{|c|}{ Left Lung } \\
\hline & \multicolumn{3}{|c|}{ Upper } & \multicolumn{3}{|c|}{ Middle } & \multicolumn{3}{|c|}{ Lower } & \multicolumn{3}{|c|}{ Upper } & \multicolumn{3}{|c|}{ Middle } & \multicolumn{3}{|c|}{ Lower } \\
\hline & 0 & $0^{+}$ & $\mathrm{I}$ & 0 & $0^{+}$ & I & 0 & $0^{+}$ & I & 0 & $0^{+}$ & I & 0 & $0^{+}$ & I & 0 & $0^{+}$ & I \\
\hline Radiography & 0 & 0 & 0 & 0 & 0 & 0 & 9 & 20 & 39 & 0 & 0 & 0 & 0 & 0 & 0 & 9 & 20 & 39 \\
\hline HRCT & 0 & 1 & 3 & 0 & 2 & 4 & 11 & 22 & 41 & 0 & 0 & 2 & 0 & 2 & 4 & 11 & 22 & 40 \\
\hline
\end{tabular}

The numbers of small opacities in HRCT scans were significantly higher than those on radiography in all lung zones. $\chi^{2}=15.783$, $p=0.007$.

Table 3. Comparison of radiography and HRCT scans of numbers of lung zones by profusion of small opacities

\begin{tabular}{|c|c|c|c|c|c|c|c|c|c|}
\hline \multirow[t]{3}{*}{ Group } & \multicolumn{9}{|c|}{ Profusion } \\
\hline & \multicolumn{3}{|c|}{0} & \multicolumn{3}{|c|}{$0-1$} & \multicolumn{3}{|c|}{1} \\
\hline & 0 & $0^{+}$ & I & 0 & $0^{+}$ & I & 0 & $0+$ & I \\
\hline Radiography & 180 & 135 & 120 & 0 & 30 & 0 & 0 & 0 & 75 \\
\hline HRCT & 172 & 131 & 105 & 4 & 30 & 0 & 0 & 19 & 179 \\
\hline
\end{tabular}

$\chi^{2}=4.173, p=0.124$.

in HRCT scans were significantly higher than those in radiography of $0^{+}$and $I$ period silicosis (emphysema $0^{+}: \chi^{2}=9.60, p=0.002$; emphysema I: $\chi^{2}=22.26, p=0.000$; bulla $0^{+}: \chi^{2}=4.32, p=0.038$; bulla I: $\chi^{2}=10.76, p=0.001$ ).

Thirty-two cases of abnormal pleural changes were detected in HRCT scans. Only 5 cases of pleural thickening were detected in radiography. The detection rate of pleural thickening in HRCT scans was significantly higher than that in radiography of $0^{+}$and I period silicosis $\left(0: p=0.011 ; 0^{+}: \chi^{2}=6.67, p=0.010 ; \mathrm{I}: \chi^{2}=11.43, p=0.001\right)$.

Calcification of the hilar and mediastinal lymph node was detected in 41 subjects' HRCT scans. However, these changes could only be detected on 10 subjects' radiographs. The detection rate of calcification of the lymph node in HRCT scans was significantly higher than that in radiography of $0^{+}$and $\mathrm{I}$ period silicosis $\left(0^{+}: \chi^{2}=19.20, p=0.000 ; \mathrm{I}: \chi^{2}=8.30, p=0.004\right)$.

Eight cases of pulmonary tuberculosis and 3 cases of cavitation were detected in HRCT scans. Six cases of pulmonary tuberculosis and 1 case of cavitation were detected in radiographs. No statistical significances were found between radiography and HRCT in the detection of pulmonary tuberculosis and cavity.

\section{Discussion}

The diagnosis of silicosis is based mainly on the radiological findings of workers exposed to dust causing lung fibrosis. However, on radiograms many parenchymal structures overlap, which limits the sensitivity and specificity of the method. Difficulties in accurate interpretation of conventional radiograms in silicosis also result from their relatively low resolution. This study aimed to assess the value and usefulness of HRCT in the diagnostics of small opacities and complications of silicosis, compared to conventional radiograms.

Radiography is not perfect since it cannot reveal early intraparenchymatous changes and does not fully reconstruct their extent. Hence, a normal picture of conventional radiography in people exposed to dust does not exclude the presence of silicosis. Due to individual sensitivity to dust, and connected with this, individually variable progression of pulmonary changes and their apparent regression with age in some cases, there is the need for techniques for their visualization, especially for early changes ${ }^{13)}$. These would enable recognition at the subclinical stage and prognoses of the course of the disease. The extent of silicosis in HRCT is determined by pulmonary fields with increased density corresponding to nodular opacities ${ }^{10)}$. Parenchymatous changes revealed in HRCT are often invisible, poorly visible, or doubtful in conventional radiography ${ }^{14)}$. HRCT can reveal small opacities earlier than conventional radiograms and assessment of their concentration is more precise ${ }^{15)}$. HRCT sections detected small and multivocal opacities than more accurately and earlier radiograms ${ }^{16)}$. HRCT also allows assessment of the relationship of opacities to the pulmonary lobule. Visualisation of early and tiny nodular changes is also possible as a result of reduced overlapping of parenchymatous structures ${ }^{17,18)}$.

In this study, 8, 5 and 6 subjects were respectively diagnosed as $0^{+}$, I or $\mathrm{I}^{+}$period silicosis based on HRCT 
of 90 subjects whose original diagnoses were nonsilicosis, $0^{+}$or I period silicosis based on radiography. The numbers of small opacities in HRCT scans were significantly higher than those on radiography in all lung zones. The comparison of radiography and HRCT scans of complications of silicosis demonstrates the greater value of the latter in the early diagnosis of silicosis. HRCT may be more sensitive than radiography in detecting small opacities of mid-out zones of the lung. The comparison of radiography and HRCT scans of small opacities in silicosis subjects demonstrated the greater potential of the latter in the early diagnosis of silicosis, as well as in the detection of additional X-ray findings (pleural thickenings, bulla forming, emphysema, etc.). HRCT has potential diagnostic value for silicosis, especially in the early stage of silicosis. It provides significant additional information for patients with silicosis and enables differentiation of potentially malignant alterations. It also reveals reticular micronodular forms of silicosis, manifested by irregular opacities and single small opacities ${ }^{19}$.

HRCT is not currently accepted as a diagnostic tool for the detection of pneumoconiosis, because the examination cost of HRCT is expensive and there is current diagnosis standard of silicosis based on HRCT. Because HRCT scans might be more sensitive than chest radiography in detecting lung parenchymal changes suggestive of pneumoconiosis and early confluence of small opacities in the lung, HRCT scans should be considered for the better and earlier diagnosis of pneumoconiosis.

\section{References}

1) Akira M, Yamamoto S, Inoue Y and Sakatani M: Highresolution $\mathrm{CT}$ of asbestosis and idiopathic pulmonary fibrosis. Am J Roentgenol 181, 163-169 (2003)

2) Bergin CJ, Müller NL, Vedal S and Chan-Yeung M: CT in silicosis: correlation with plain films and pulmonary function tests. Am J Roentgenol 146, 477483 (1986)

3) Remy-Jardin M, Beuscart R, Sault MC, Marquette CH and Remy J: Subpleural micronodules in diffuse infiltrative lung diseases: evaluation with thin-section CT scans. Radiology 177, 133-139 (1990)

4) Ministry of Health of PR China. Diagnostic standard of pneumoconiosis. GBZ70-2002. 2002.

5) Tonori Y, Niitsuya M, Sato T, Sugiura Y, Miyake H and Aizawa Y: Relationship between Chest X-ray Finds and Pulmonary Function Tests in Dust Workers. Ind Health 43, 256-266 (2005)

6) Kim KI, Kim CW, Lee MK, Lee KS, Park CK, Choi SJ and Kim JG: Imaging of occupational lung disease.
Radiographics 21, 1371-1391 (2001)

7) Stark P, Jacobson F and Shaffer K: Standard imaging in silicosis and coal worker's pneumoconiosis. Radiol Clin North Am 30, 1147-1154 (1992)

8) Akira M: Uncommon pneumoconioses: CT and pathologic findings. Radiology 197, 403-409 (1995)

9) Zlomaniec G, Mosiewicz J, Zlomaniec J and Krupski W: HRCT examinations in the course of silicosis. Ann Univ Mariae Curie Sklodowska [Med] 56, 157-163 (2001)

10) Mosiewicz J, Myśliński W, Zlomaniec G, CzabakGarbacz R, Krupski W and Dzida G: Diagnostic value of high resolution computed tomography in the assessment of nodular changes in pneumoconiosis in foundry workers in Lublin. Ann Agric Environ Med 11, 279-284 (2004)

11) Ferreira AS, Moreira VB, Ricardo HM, Coutinho R, Gabetto JM and Marchiori E: Progressive massive fibrosis in silica-exposed workers. High-resolution computed tomography findings. J Bras Pneumol 32, 523-528 (2006)

12) Petrova E, Petkov D, Shoshkov P and Nachev C: The diagnostic value of conventional $\mathrm{X}$-ray examination of the lungs in comparison with high-resolution computed tomography (HRCT), isotope perfusion scintigraphy and the diffusion capacity in patients with pneumoconiosis. Int J Occup Med Environ Health 8, 231-238 (1995)

13) Bergin R, Ostiguy G, Fillion R and Colman N: Computed tomography scan in the early detection of silicosis. Am Rev Respir Dis 144, 697-705 (1991)

14) Meziane MA, Hruban RH, Zerhouni EA, Wheeler PS, Khouri NF, Fishman EK, Hutchins GM and Siegelman SS: High-resolution CT of the lung parenchyma with pathologic correlation. Radiographics 8, 27-54 (1988)

15) Lee KS, Kim TS, Han J, Hwang JH, Yoon JH, Kim Y and Yoo SY: Diffuse micronodular lung disease: HRCT and pathologic findings. J Comput Assist Tomogr 23, 99-106 (1999)

16) Kraus T, Raithel HJ and Hering KG: Evaluation and classification of high-resolution computed tomographic findings in patients with pneumoconiosis. Int Arch Occup Environ Health 68, 249-254 (1996)

17) Remy-jardin M, Degreef JM, Beuscart R, Voisin C and Remy J: Coal worker's pneumoconiosis: CT assessment in exposed worker's and correlation with radiographic findings. Radiology 177, 363-371 (1990)

18) Remy-jardin M, Remy J, Farre I and Marguette $\mathrm{CH}$ : Computed tomographic evaluation of silicosis and coal worker's pneumoconiosis. Radiol Clin North Am 30, 1155-1176 (1992)

19) Schaefer-Prokop C, Prokop M, Fleischmann D and Herold C: High-resolution CT of diffuse interstitial lung disease: key findings in common disorders. Eur Radiol 11, 373-392 (2001) 